\title{
Ultrasensitive Detection and Quantification of Acidic Disaccharides Using Capillary Electrophoresis and Quantum Dot-Based Fluorescence Resonance Energy Transfer
}

Yuqing Chang, ${ }^{\dagger}$ Chao Cai, ${ }^{\dagger}$ Lingyun Li, $^{\dagger}$ Jianjun Miao, ${ }^{\ddagger}$ Ebru Ucakturk, ${ }^{\dagger, \perp}$ Guoyun Li, ${ }^{\dagger, \nabla}$ Mellisa Ly, $^{\dagger}$ and Robert J. Linhardt $*, \dagger, \uparrow, \S, \|$

${ }^{\dagger}$ Department of Chemistry and Chemical Biology, ${ }^{\ddagger}$ Chemical and Biological Engineering, ${ }^{\S}$ Biology, and "Biomedical Engineering, Center for Biotechnology and Interdisciplinary Studies, Rensselaer Polytechnic Institute, Troy, New York 12180, United States

${ }^{\perp}$ Department of Analytical Chemistry, Faculty of Pharmacy, Hacettepe University, Sihhiye, Ankara, 06100, Turkey

${ }^{\nabla}$ College of Food Science and Technology, Ocean University of China, Qingdao, Shandong 266003, China

Supporting Information

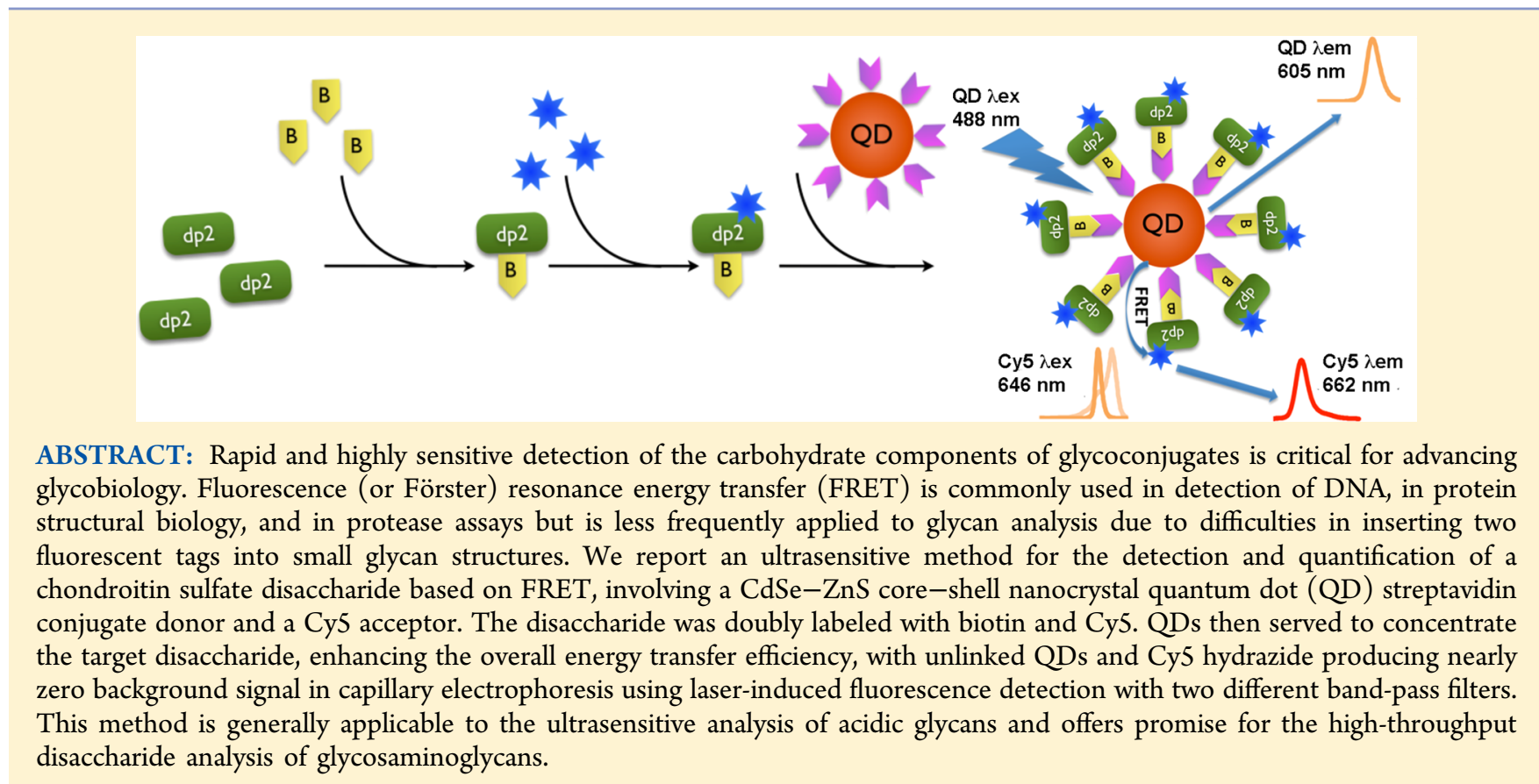

$\mathrm{F}$

luorescence (or Föster) resonance energy transfer (FRET)

- is a process involving the transfer of energy from donor fluorophore to acceptor fluorophore when the distance between the donor and the acceptor is smaller than a critical radius, known as the Förster radius $\left(R_{0}\right)$. This leads to a reduction in the donor's emission and excited state lifetime and an increase in the acceptor's emission intensity. ${ }^{1}$ FRET is widely applied in measuring protein conformational changes ${ }^{2}$ and in enzyme activity assays. ${ }^{3}$ But FRET has infrequently been applied to carbohydrate analysis due to the general lack of appropriately spaced, reactive sites on glycans for the introduction of two fluorescent tags. Acidic glycans, such as glycosaminoglycan-derived disaccharides, offer an attractive FRET target as they have both a single reactive hemiacetal reducing end and a single nonreducing end carboxyl group. ${ }^{4}$

FRET requires fluorescent molecules in close proximity in the range of $0-2 R_{0}$. Even at very high concentrations noninteracting donors and acceptors do not undergo FRET. This is considered an advantage of FRET, as excess donor and/ or acceptor fluorophores are often used to promote nonbonded interactions of donor and acceptor. Since this large excess of unbound fluorophore should not add to FRET, a purification step, typically required in most fluorescence experiments, is unnecessary. While FRET is primarily detected using separation-free spectroscopic and microscopic imaging techniques, ${ }^{5-10}$ in practice, these detection methods can lead to misleading or even meaningless results. ${ }^{11}$ The major factor causing inaccuracy in calculating FRET efficiency is crosstalk between the two fluorophores. Not only can the acceptor be excited with the light selected to excite the donor but some of

Received: July 20, 2013

Accepted: August 28, 2013

Published: August 28, 2013 

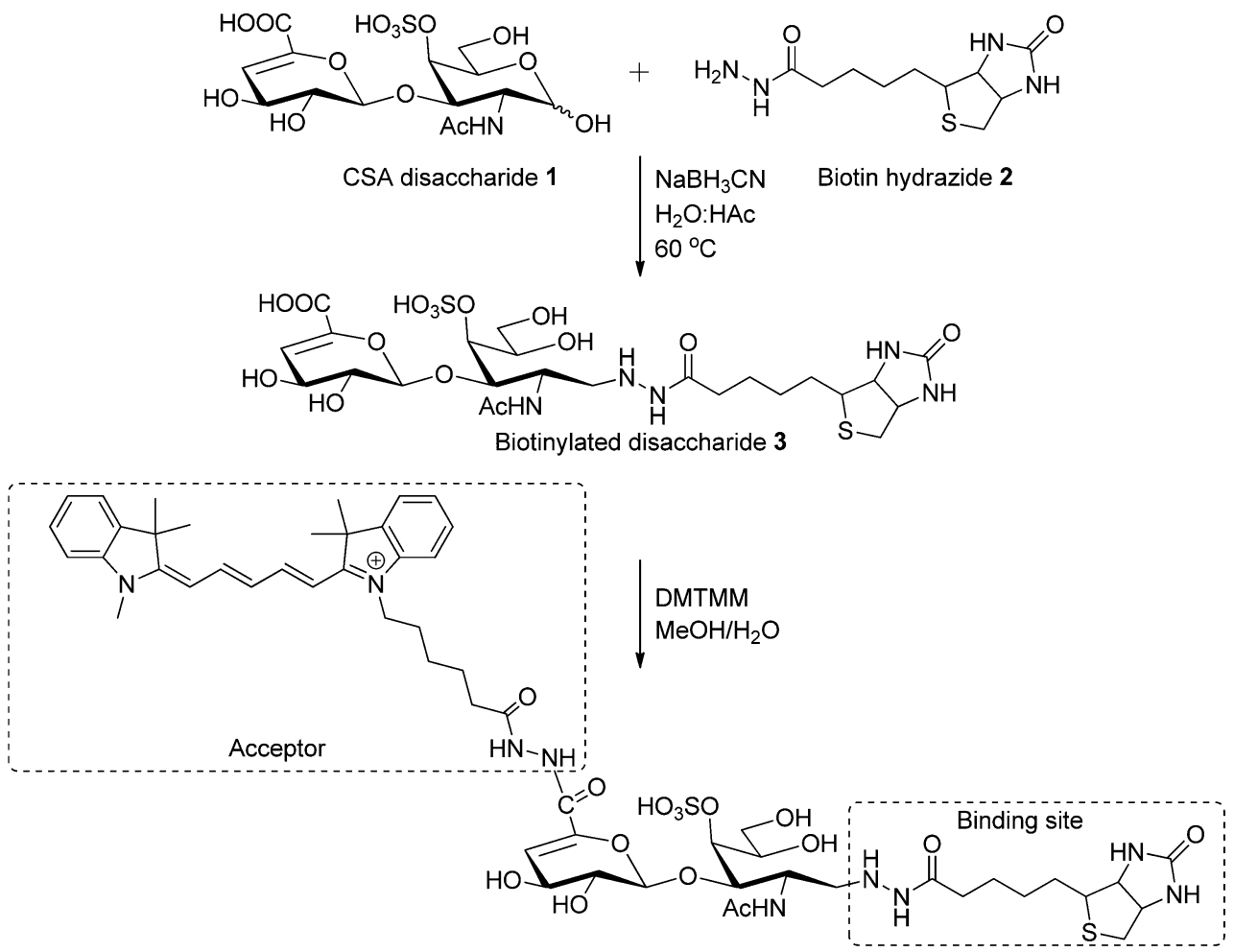

Biotinylated and Cy5 labeled disaccharide 4

Figure 1. Synthesis pathway is shown for the Cy5-disaccharide-biotin FRET acceptor.

the detected fluorescence can also come from the excited donor. Quantum dots (QDs) have emerged as particularly effective FRET donors due to their narrow emission spectra. While QDs can decrease excitation crosstalk, they cannot eliminate spectral crosstalk in the detected signal. One approach for completely eliminating crosstalk is to combine a separation method with FRET analysis.

Capillary electrophoresis (CE) is a powerful high-resolution method capable of separating QDs and their conjugates. ${ }^{12,13}$ In the commonly used capillary zone electrophoresis (CZE), a bare fused-silica capillary can separate QDs with different charge-to-size ratios under optimized conditions. While capillary gel electrophoresis ${ }^{14}$ has also been applied to improve resolution of QDs and QD-bioconjugate mixtures, a decrease in detection sensitivity often results. Several groups ${ }^{13,15,16}$ have reported the CE-based separation of QDs and their bioconjugates. FRET has been detected between water-soluble CdTe QD donor and $632 \mathrm{~nm}$ emitting CdSe/ZnS QD acceptor covalently conjugated with antibodies using Capillary electrophoresis laser-induced fluorescence (CE-LIF). ${ }^{17}$ FRET has also been applied between QDs donor and Cy5 acceptor bound to a polypeptide for measuring protease activity using $\mathrm{CE}$ as separation tool for bound and unbound QDs. ${ }^{1,3,5}$ However, the separation of QDs and $\mathrm{QD}$-disaccharide conjugate is particularly challenging because the relatively small size of a disaccharide and the challenges associated with the introduction of donor and acceptor fluorophores. In the current study we have prepared a disaccharide FRET complex and optimized its separation by $\mathrm{CE}$ and its detection by LIF.

\section{EXPERIMENTAL SECTION}

Materials. All the chemicals and materials used in the experiment were of analytical grade unless otherwise indicated.
QD-605 streptavidin conjugate $\left(\mathrm{QD}_{\mathrm{SA}}\right)$ and incubation buffer were purchased from Invitrogen Inc., U.S.A. The conjugates were received as $1 \mu \mathrm{M}$ solution in a $\mathrm{pH} 8.3$ buffer composed of $50 \mathrm{mM}$ borate, $0.5 \%$ bovine serum albumin (BSA), and $0.05 \%$ sodium azide (preservative). QDs were characterized by transmission electron microscopy (TEM) upon arrival (see Supporting Information Figure S1). Cy5 hydrazide was purchased from Lumiprobe Co., U.S.A. Chondroitin sulfate (CS)-A, from bovine trachea, was purchased from Celsus Laboratories, Cincinnati, OH. Chondroitin lyases $\mathrm{ABC}$ and ACII were purchased from Associates of Cape Cod, Inc., East Falmouth, MA. Biotin hydrazide, sodium cyanoborohydride $\left(\mathrm{NaBH}_{3} \mathrm{CN}\right)$, methanol $(\mathrm{MeOH})$, dimethyl sulfoxide (DMSO), glacial acetic acid (HAc), N-methylmorpholine (NMM), 2-chloro-4,6-dimethoxy-1,3,5-triazine (CDMT), and fluorescein isothiocyanate isomer I (FITC) were purchased from Sigma-Aldrich, St. Louis, MO.

Biotinylation of Chondroitin Sulfate Disaccharide. CS disaccharide 1 (Figure 1) was prepared from CS-A by exhaustive chondroitin lyase treatment and purified as previously described. ${ }^{4}$ This monosulfated chondroitin disaccharide $\mathbf{1}$ was selected as a model glycosaminoglycan disaccharide for double labeling, structural characterization, and for high-sensitivity FRET detection. Biotin hydrazide $\mathbf{2}$ was used for CS disaccharide 1 biotinylation. The CS disaccharide 1 (5.0 mg, $12.4 \mathrm{mmol})$ was dissolved in a solution of $\mathrm{H}_{2} \mathrm{O}(1.7$ $\mathrm{mL})$ and $\mathrm{AcOH}(0.3 \mathrm{~mL}) . \mathrm{NaBH}_{3} \mathrm{CN}(1.2 \mathrm{mg}, 18.6 \mathrm{mmol})$ and biotin hydrazide $2(4.8 \mathrm{mg}, 18.6 \mathrm{mmol})$ were added to the reaction mixture. The reaction was stirred at $60{ }^{\circ} \mathrm{C}$ for 3 days. On each day, additional portions of $\mathrm{NaBH}_{3} \mathrm{CN}(1.2 \mathrm{mg}, 18.6$ $\mathrm{mmol})$ and biotin hydrazide $(4.8 \mathrm{mg}, 18.6 \mathrm{mmol})$ were added. The reaction mixture was loaded onto a BioGel P2 column (2.5 $\mathrm{cm} \times 65 \mathrm{~cm})$ and eluted with $\mathrm{H}_{2} \mathrm{O}$. Fractions were collected, 
A
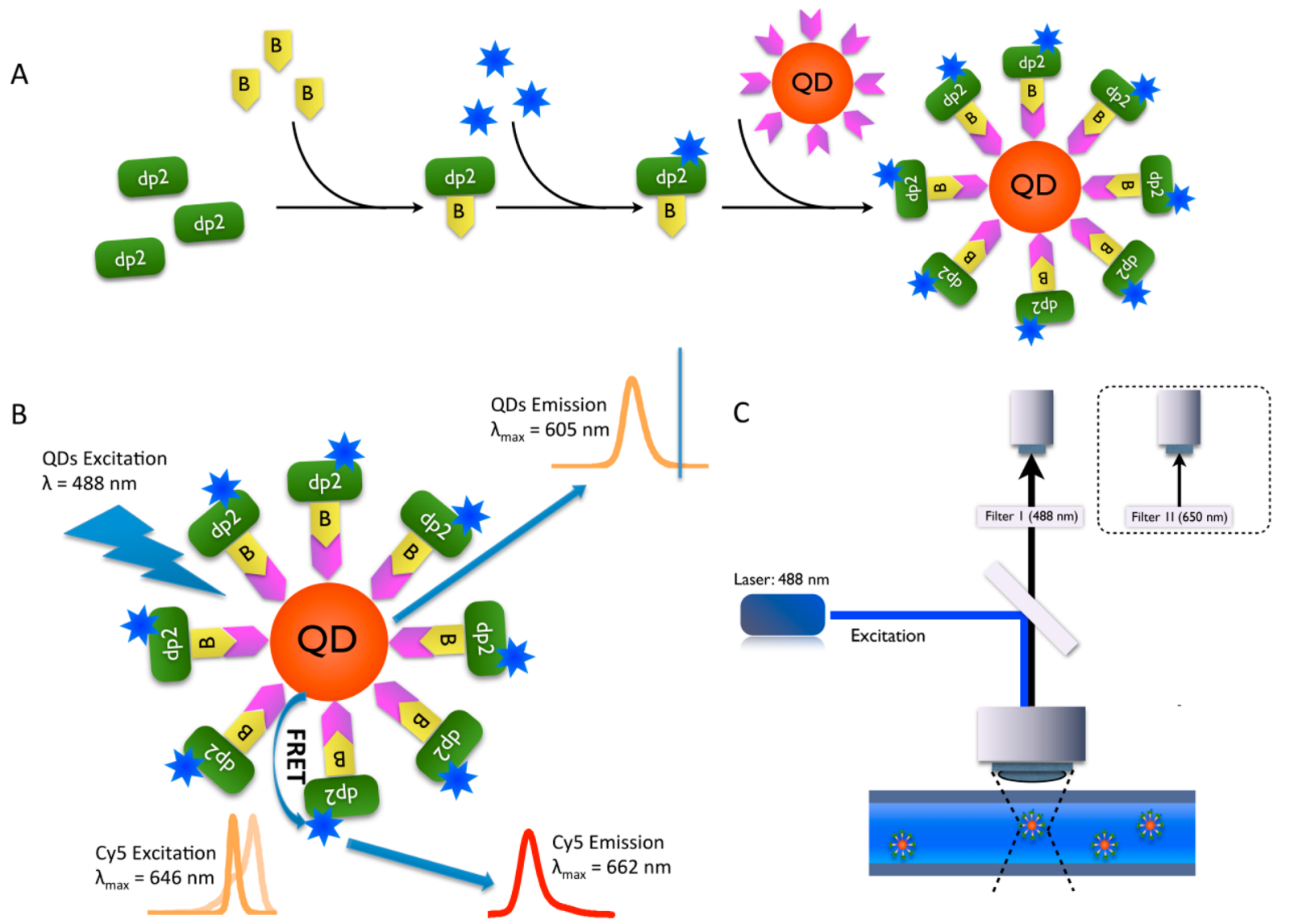

Figure 2. Scheme of the FRET system construction in this study. (A) Conjugation scheme of the FRET system. Disaccharide (degree of polymerization ( $\mathrm{dp}$ ) 2, green) is biotinylated (B, yellow), then coupled to the FRET acceptor, Cy5 hydrazide (blue star). Incubation at room temperature and in the dark of $\mathrm{Cy} 5-\mathrm{dp2}-$ biotin complex 4 to $\mathrm{QD}$ streptavidin conjugate $\left(\mathrm{QD}_{\mathrm{SA}}\right.$, red and purple) forms the FRET complex 4$\mathrm{QD}_{\mathrm{SA}}$. (B) The FRET donor, QD, is excited with a laser at $488 \mathrm{~nm}$; because Cy5 dye, located on the same disaccharide, is close, FRET occurs and $\mathrm{Cy} 5$ is excited by emission from the QD, and the emission of Cy5 is then detected. (C) CE-LIF instrumental setup for FRET detection. A $488 \mathrm{~nm}$ (filter I, donor-acceptor channel) and $650 \mathrm{~nm}$ (filter II, acceptor channel) band-pass filter was used for FRET detection.

and those containing the product as determined by thin-layer chromatography (TLC) $\left(n-\mathrm{BuOH} / \mathrm{AcOH} / \mathrm{H}_{2} \mathrm{O}=2 / 2 / 1\right)$ were combined and freeze-dried to afford the biotinylated disaccharide 3 as a white powder.

Coupling of Cy5 to Biotinylated Disaccharide. 4-(4,6Dimethoxy-1,3,5-triazin-2-yl)-4-methylmorpholinium chloride (DMTMM) was prepared by adding NMM (2.02 g, 20 mmol) to a solution of CDMT $(3.86 \mathrm{~g}, 22 \mathrm{mmol})$ in THF (60 $\mathrm{mL}$ ) at room temperature. A white solid appeared within several minutes. After stirring for $30 \mathrm{~min}$ at room temperature, the solid was collected by suction and washed with THF and dried to give DMTMM (100\%). Although the DMTMM was of high purity, it was recrystallized from methanol and diethyl ether before using. Biotinylated disaccharide $3(1 \mathrm{mg})$ was dissolved in methanol-water $(9: 1,5 \mathrm{~mL})$ together with the Cy5 hydrazide (1.2 equiv), and the mixture was stirred at room temperature for $10 \mathrm{~min}$. Recrystallized DMTMM (1.5 equiv) was then added, and the reaction mixture was stirred at room temperature until the reaction was complete $(5-14 \mathrm{~h})$. The solvent was removed under reduced pressure, and the residue coevaporated with absolute ethanol affording the biotinylated, Cy5-labeled disaccharide 4.

Complexation of QD-605 Streptavidin QD $_{S A}$ with Biotinylated Cy5-Labeled Disaccharide 4. Complexation of QD-605 streptavidin $\left(\mathrm{QD}_{\mathrm{SA}}\right)$ with biotinylated Cy5-labeled disaccharide 4 was carried out in $\mathrm{QD}$ incubation buffer provided by Invitrogen Inc. Before conjugation, $\mathrm{QD}_{\mathrm{SA}}$ was centrifuged at $5000 \mathrm{~g}$ for $3 \mathrm{~min}$; any precipitate was discarded before reaction. Amounts of 32, 20, 16, 12, 8, 4, 2, and 1 pmol of 4 and $1 \mu \mathrm{L}$ of $\mathrm{QD}_{\mathrm{SA}}(1 \mu \mathrm{M})$ were added to incubation buffer, respectively. The final concentration of $\mathrm{QD}_{\mathrm{SA}}$ is $10 \mathrm{nM}$. The mixtures were left in dark for $5 \mathrm{~h}$ to complete the conjugation. Samples were diluted with incubation buffer to desired concentration for CE-LIF analyses of $4-\mathrm{QD}_{\mathrm{SA}}$ complex.

Instrumentation. CE analyses were carried out on an Agilent G1600 high-performance CE system coupled with a ZETALIF (Picometrics, France) detector $\left(\lambda_{\mathrm{ex}}=488 \mathrm{~nm}\right)$. Resolution and analysis were performed on an uncoated fusedsilica capillary column $(25,50$, or $75 \mu \mathrm{m}$ i.d., indicated in each experiment) at $25{ }^{\circ} \mathrm{C}$, using $50 \mathrm{mM}$ carbonate buffer, $\mathrm{pH} 9.0$ (unless otherwise indicated), at different voltages, as shown in figures, normal polarity. New capillary was treated with $\mathrm{MeOH}$, $1 \mathrm{M} \mathrm{HCl}, 1 \mathrm{M} \mathrm{NaOH}, 0.1 \mathrm{M} \mathrm{NaOH}$, water, and operating buffer, until the baseline got constant. Between each run, the capillary was flushed with $0.1 \mathrm{M} \mathrm{NaOH}$ (3 min), HPLC grade water $(3 \mathrm{~min})$, and operating buffer $(5 \mathrm{~min})$. The operating buffer was filtrated through a $0.2 \mu \mathrm{m}$ membrane filter. All solutions were degassed. Samples were introduced using the pressure mode $(50 \mathrm{mbar} \times 5 \mathrm{~s})$ at the anode. The emission filters of 488 and $650 \mathrm{~nm}$ band-pass were also purchased from ZETALIF (Picometrics, France). Each time after switching the 
filter, an optical performance optimization was performed with flushing the capillary with $10^{-6} \mathrm{M}$ FITC.

Transmission electron microscopy was performed with a Philips CM12 (Eindhoven, Amsterdam, Netherlands) at an accelerating voltage of $120 \mathrm{kV}$ in bright-field mode. Dispersed quantum dots on 400 mesh TEM grids were obtained by adding one drop of diluted aqueous quantum dots solution onto a carbon-coated TEM copper grid and allowing solvent to evaporate, then further drying in a vacuum oven for $2 \mathrm{~h}$.

\section{RESULTS}

Design and Synthesis of the QD/Cy5-DisaccharideBiotin as FRET Acceptor. The FRET complex and its assembly is shown in Figure 2A. A commercially available $\mathrm{CdSe}-\mathrm{ZnS}$ core-shell nanocrystal streptavidin conjugate $\left(\mathrm{QD}_{\mathrm{SA}}\right)$ with a $15-20 \mathrm{~nm}$ diameter (Supporting Information Figure S1) was chosen as FRET donor and Cy5 hydrazide was selected as FRET acceptor (Figure 1). The Cy5 hydrazide FRET acceptor was coupled to a chondroitin sulfate derived disaccharide that had been biotinylated in a high yield (80$90 \%$ ) by reductive amination reaction (Figures 1 and $2 \mathrm{~A}$ ). ${ }^{18}$

The selection of $\mathrm{QD}_{\mathrm{SA}}$ and $\mathrm{Cy} 5$ as FRET pair (Figure 2B) allows the steady-state fluorescence detection by a CE-LIF system equipped with a $488 \mathrm{~nm}$ argon ion excitation laser and two different band-pass filters (488 and $650 \mathrm{~nm}$ cutoff) (Figure $2 \mathrm{C})$. The use of two filters with different cutoff wavelengths can distinguish the fluorescence coming from donor and acceptor. Using the $488 \mathrm{~nm}$ cutoff filter, fluorescence from $\mathrm{QD}_{\mathrm{SA}}$ at 605 $\mathrm{nm}$ and fluorescence from $\mathrm{Cy} 5$ (resulting from energy transferred by the QD) at $662 \mathrm{~nm}$ are both detected. Using the $650 \mathrm{~nm}$ cutoff filter, the fluorescence from $\mathrm{QD}_{\mathrm{SA}}$ was nearly completely filtered out; as a result, only the fluorescence emission from Cy5 at $662 \mathrm{~nm}$ (coming from energy transferred by the $\mathrm{QD}$ ) is detected. The negative controls, unassembled components, $\mathrm{Cy} 5$, and $\mathrm{QD}_{\mathrm{SA}}$, were also tested and produced almost no background fluorescence.

As a FRET model for disaccharide analysis, a chondroitin sulfate derived disaccharide $\mathbf{1}$ was biotinylated by reductive animation (Figure 1) so that it could be bound to the $\mathrm{QD}_{\mathrm{SA}}$ through a strong noncovalent streptavidin-biotin interaction (Figure 2A). The biotinylated disaccharide 2 was next covalently conjugated to Cy5 hydrazide using a carbodiimide reaction. Because a double bond at the nonreducing end of this disaccharide is produced in the enzymatic digestion of chondroitin sulfate, conventional carbodiimide coupling did not effectively promote the reaction between the unsaturated carboxyl group on biotinylated disaccharide 3 and Cy5 hydrazide. Instead, the more reactive $\mathrm{DMTMM}^{19}$ was used to facilitate this coupling reaction, affording the biotinylated and Cy5-labeled disaccharide 4. The last step is to assemble $\mathrm{QD}_{\mathrm{SA}}$ to biotinylated and Cy5-labeled disaccharide 4 to form a FRET pair 4- $\mathrm{QD}_{\mathrm{SA}}$ (Figure 2A). The $488 \mathrm{~nm}$ laser light (Figure 2C) excites the $\mathrm{QD}$, resulting in $\mathrm{QD}$ emission, excitation of Cy5, and emission of Cy5 FRET, allowing detection of the disaccharide analyte (Figure 2B).

CZE Separation of QDs and QD-Disaccharide Complex. Conditions for the $\mathrm{CE}$ separation of $\mathrm{QD}_{\mathrm{SA}}$ from its $4-\mathrm{QD}_{\mathrm{SA}}$ FRET complex was next examined. CZE of $\mathrm{QD}_{\mathrm{SA}}$ in a bare fused-silica $75 \mu \mathrm{m}$ internal diameter (i.d.) capillary using $50 \mathrm{mM}$ sodium borate, sodium carbonate, Trishydrochloride, and sodium phosphate buffers at $\mathrm{pH} 9.0$ showed that the sodium carbonate buffer gave narrowest peak width (Supporting Information Figure S2). Furthermore, 18 injections of QDs in sodium carbonate buffer showed excellent repeatability and a small relative standard deviation (RSD) of migration time $(2.1 \%)$ and peak areas $(2.8 \%)$.

The separation of $\mathrm{QD}_{\mathrm{SA}}$ from $4-\mathrm{QD}_{\mathrm{SA}}$ complex by CZE was examined on a $75 \mu \mathrm{m}$ i.d. capillary at $\mathrm{pH} 8-11$. Disaccharide 4 was incubated with $\mathrm{QD}_{\mathrm{SA}}$ in the dark for $5 \mathrm{~h}$, and CZE was performed on a mixture of $\mathrm{QD}_{\mathrm{SA}}$ and $4-\mathrm{QD}_{\mathrm{SA}}$ complex (Supporting Information Figure S3). Complete separation of $\mathrm{QD}_{\mathrm{SA}}$ and $4-\mathrm{QD}_{\mathrm{SA}}$ complex was unsuccessful. Interestingly, the elution order of $\mathrm{QD}_{\mathrm{SA}}$ and $4-\mathrm{QD}_{\mathrm{SA}}$ complex reversed when the $\mathrm{pH}$ of the running buffer reached 10 . This is attributed to the $\mathrm{pH}$ dependence of the negatively charged residues in the streptavidin coating on $\mathrm{QD}_{\mathrm{SA}}$. Peak broadening at $\mathrm{pH} 10.0-$ 11.0 suggested that neither $\mathrm{QD}_{\mathrm{SA}}$ nor $4-\mathrm{QD}_{\mathrm{SA}}$ complex were stable in buffer higher than $\mathrm{pH} 9.0$; thus, $\mathrm{pH} 9.0$ was selected for subsequent experiments.

Next, the effect of voltage of $8-16 \mathrm{kV}$ on the separation of $\mathrm{QD}_{\mathrm{SA}}$ and 4- $\mathrm{QD}_{\mathrm{SA}}$ complex was examined (Supporting Information Figure S4). As separation voltage decreased, migration time of both analytes increased, peak broadening was observed, and resolution did not significantly increase; thus, $16 \mathrm{kV}$ affording the fastest migration time was selected for subsequent experiments.

The utilization of polymeric additives as sieving medium can improve the $\mathrm{CE}$ separation of analytes, particularly biomolecules. ${ }^{16,20} \mathrm{CE}$ analysis of a mixture of $\mathrm{QD}_{\mathrm{SA}}$ and $4-\mathrm{QD}_{\mathrm{SA}}$ complex was carried out using various poly(ethylene glycol) (PEG) $(20 \mathrm{kDa})$ concentrations $(0-4 \%)$ as sieving medium (Supporting Information Figure S5). At 2\% PEG solution gave an optimal separation of $\mathrm{QD}-\mathrm{QD}_{\mathrm{SA}}$ and $4-\mathrm{QD}_{\mathrm{SA}}$ complex. We concluded that the enhanced resolution justified the slight decrease in fluorescence intensity observed, and thus, 2\% PEG was included in subsequent experiments.

Finally, an ultrathin capillary $(25 \mu \mathrm{m}$ i.d., $50 \mathrm{~cm}$ effective length) was used for the $\mathrm{CE}$ separation of $\mathrm{QD}_{\mathrm{SA}}$ and $4-\mathrm{QD}_{\mathrm{SA}}$ complex in $50 \mu \mathrm{m}$ sodium bicarbonate ( $\mathrm{pH}$ 9) containing $2 \%$ PEG at $16 \mathrm{kV}$ to afford optimal separation (Supporting Information Figure S6).

FRET Detection of $4-\mathrm{QD}_{\mathrm{SA}}$ Complex on CE-LIF. Reaction mixtures with donor-acceptor ratio of 1:20 with a final $\mathrm{QD}_{\mathrm{SA}}$ concentration of $10 \mathrm{nM}$ were incubated for $1-6 \mathrm{~h}$ and then analyzed by CE-LIF to determine optimal time for the formation of $4-\mathrm{QD}_{\mathrm{SA}}$ complex. Mixtures of $\mathrm{QD}_{\mathrm{SA}}$ and $\mathrm{Cy} 5$ hydrazide of the same molar ratio were used as a negative control. In this study, the percentage of $4-\mathrm{QD}_{\mathrm{SA}}$ FRET complex in the mixture was approximately calculated by

$$
\begin{aligned}
& \text { percentage FRET complex } \\
& =\frac{\beta A_{4-\mathrm{QDSA} \text { complex }}}{\beta A_{4-\mathrm{QDSA} \text { complex }}+A_{\mathrm{QDSA}}} \times 100
\end{aligned}
$$

where $A_{4-\mathrm{QDSA}}$ complex is the peak area of the $4-\mathrm{QD}_{\mathrm{SA}}$ FRET complex, and $A_{\mathrm{QDSA}}$ is the peak area of $\mathrm{QD}_{\mathrm{SA}}$. The relative value of quantum yield was $\beta=2.5$ ( $\beta$ equals 1 only when the quantum yields of two fluorophores are not significantly different).

The electrophoresis peaks of $\mathrm{QD}_{\mathrm{SA}}$ and $4-\mathrm{QD}_{\mathrm{SA}}$ FRET complex were observed in the donor-acceptor channel (488 $\mathrm{nm}$ filter), whereas in the acceptor channel (650 nm filter) only fluorescence from 4- $\mathrm{QD}_{\mathrm{SA}}$ FRET complex was observed (Supporting Information Figure S7, parts A and C). The percentage and fluorescence intensity of $4-\mathrm{QD}_{\mathrm{SA}}$ FRET complex increased as reaction time increased (Supporting 

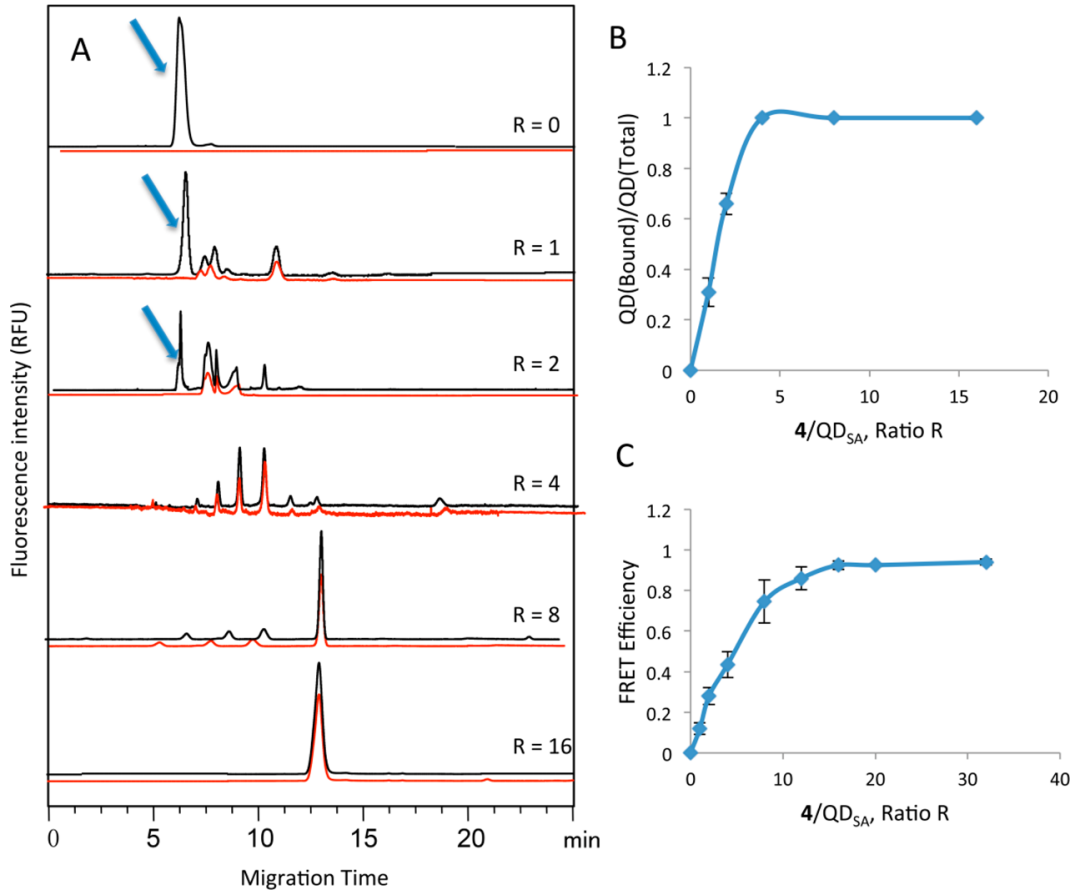

C

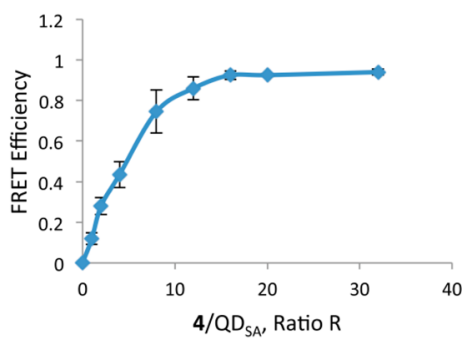

Figure 3. FRET between Cy5-labeled biotinylated disaccharide 4 and $\mathrm{QD}-605$ streptavidin conjugate $\mathrm{QD}_{\mathrm{SA}}$ at different ratios. (A) Electropherograms of complexes of 4 and $\mathrm{QD}_{\mathrm{SA}}$ at different ratios $\left(4 / \mathrm{QD}_{\mathrm{SA}}, R=0,1,2,4,8,16\right)$, monitored by CE-LIF from the donoracceptor channel (black) and acceptor channel (red). (B) The percentage of bound QDs increases with increasing $R$ and reaches $100 \%$ at an $R$ of $4 /$ 1. (C) The FRET efficiency increases as $R$ increases and reaches a saturated state at an $R$ of 16/1. The final QDs concentration in the reaction mixture was $10 \mathrm{nM}$, and the amount of 4 present was $0,1,2,4,8,16,32$ pmol, respectively.
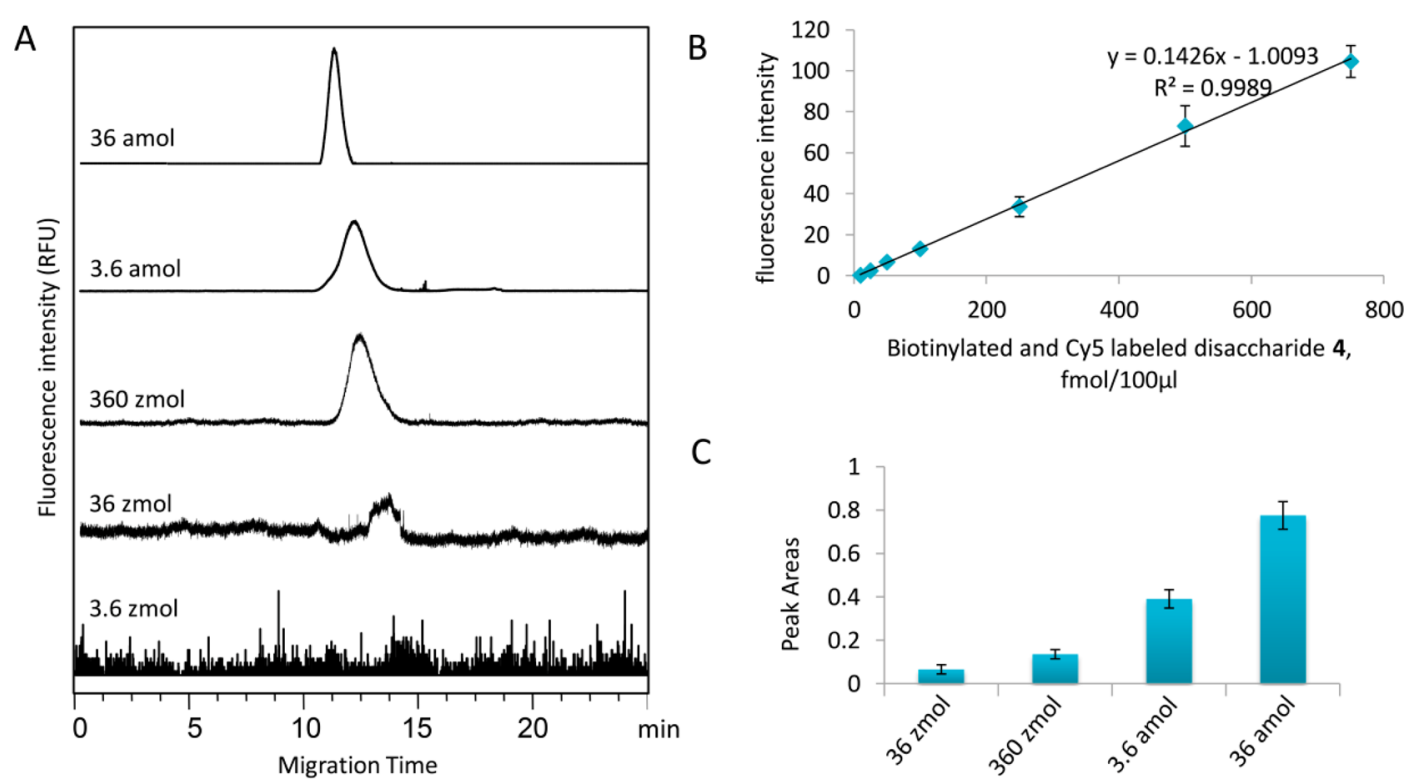

C

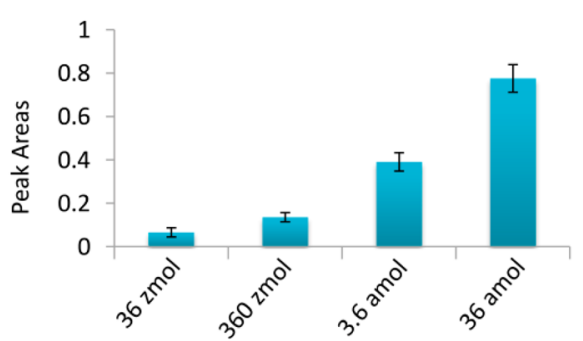

Figure 4. FRET-based disaccharide assay. (A) Electropherograms of injection of 36 amol to $3.6 \mathrm{zmol}$ of $4-\mathrm{QD}_{\mathrm{SA}}$ complex. (B) Linear equation of fluorescence intensity of the FRET complex from the acceptor channel plotting against concentration of 4- $\mathrm{QD}_{\mathrm{SA}} \mathrm{complex}$, from 10 to $750 \mathrm{fmol} / 100$ $\mu \mathrm{L}$ of solution. (C) Variance of FRET intensity from the acceptor channel at $36 \mathrm{zmol}$ to 36 amol with three parallel injections.

Information Figure S7, parts B and D). After $2 \mathrm{~h}$, the percentage of $4-\mathrm{QD}_{\mathrm{SA}}$ FRET complex reached $50 \%$, and after $4 \mathrm{~h}$ it increased to $92 \%$, and reached $\sim 100 \%$ at $5 \mathrm{~h}$. Therefore, $5 \mathrm{~h}$ was chosen as the complex formation time in subsequent experiments. In the control experiments (Supporting Information Figure S7C) with unlinked $\mathrm{QD}_{\mathrm{SA}}$ and $\mathrm{Cy} 5$ hydrazide (1:20), fluorescence signals were not detected in the acceptor channel, indicating that no crosstalk was occurring between donor and acceptor. The FRET efficiency was calculated at $\sim 85 \%$.

The concentrations of both $\mathrm{QD}_{\mathrm{SA}}$ and $4-\mathrm{QD}_{\mathrm{SA}}$ FRET complex need to be optimized to perform quantitative FRET. The optimum ratio $(R)$ of biotinylated Cy5 disaccharide to QD-650 streptavidin conjugate was examined (Figure 3A). Because $\mathrm{QD}_{\mathrm{SA}}$ is well-resolved from the $4-\mathrm{QD}_{\mathrm{SA}}$ FRET complex, an accurate calculation of FRET efficiency and binding percentage could be obtained. While saturation of $Q D$ 
binding occurred at $R=4$ (Figure 3B), multiple peaks of the FRET complex were observed until $R=16$, when FRET efficiency reached $91 \%$ and remained unchanged even at higher $R$-values (Figure 3C).

Determination of Limit of Detection. The quantitative properties of the method were tested by injecting a series of different 4- $\mathrm{QD}_{\mathrm{SA}}$ FRET complexes. A standard curve was plotted from 10 to $750 \mathrm{fmol} / 100 \mu \mathrm{L}$, a ratio of $4 / \mathrm{QD}_{\mathrm{SA}}$ was fixed at 20:1, and the peak area from the $4-\mathrm{QD}_{\mathrm{SA}}$ FRET complex from the acceptor channel was recorded and integrated (Figure 4). A limit of detection (LOD) of $36 \mathrm{zmol}$ of disaccharide (21600 molecules of 4) was obtained. Ten injections were performed to test CE repeatability at $1 \mathrm{pmol} /$ $100 \mu \mathrm{L}$; an RSD of $1.2 \%$ in peak area and $0.9 \%$ in migration time of the $4-\mathrm{QD}_{\mathrm{SA}}$ FRET complex in the acceptor channel indicates the method has excellent repeatability. Moreover, the complex was stable in the dark at room temperatures for at least 5 days and a very low variance of FRET signal in acceptor channel was observed at the low concentrations tested (Figure 4C).

\section{DISCUSSION}

FRET requires a donor having a high quantum yield, spectral overlap of the emission spectrum of donor and absorbance spectrum of acceptor, close proximity $\left(R_{0}\right)$ between donor and acceptor, and an effective detection system. Unlike organic dyes, QDs offer some advantages when serving as FRET donors. Because QDs have higher quantum yield than conventional dyes, a QDs/organic dye hybrid FRET system usually produces higher sensitivity than organic-only FRET systems. QDs also have a narrow emission spectrum, which helps to decrease the level of background fluorescence and avoid spectral crosstalk and direct acceptor excitation. ${ }^{21}$ Furthermore, QDs have longer fluorescent lifetimes, can undergo many excitation cycles, and have size-dependent excitation wavelengths, making QDs excellent candidates as FRET donors.

In this paper we designed a FRET system with a QD and an organic dye, Cy5, that, when coupled to CE-LIF detection, serves as a probe for ultrasensitive quantification. The QDCy5 FRET pair, selected for minimum spectral overlap between donor emission and acceptor absorbance, was limited through multiple streptavidin proteins on the $Q D$ surface to enrich multiple Cy5 acceptors on a single QD, thus maximizing the overall energy transfer efficiency and sensitivity. ${ }^{22}$ The QD-605 streptavidin conjugate $\left(\mathrm{QD}_{\mathrm{SA}}\right)$ has 4-10 biotin binding sites per $\mathrm{QD}$ on the surface. ${ }^{23}$ Therefore, energy transfer efficiency between $\mathrm{QD}_{\mathrm{SA}}$ and $\mathrm{Cy} 5$ was markedly enhanced.

A biotinylated disaccharide 3 serves as an extremely high affinity $\left(10^{-14} \mathrm{M}\right)$ bridge between the Cy5 acceptor and the $\mathrm{QD}_{\mathrm{SA}}$ donor. This interaction is resistant to extremes of heat, $\mathrm{pH}$, and proteolysis, making it a good candidate for CE-based separation. The Förster radius of this $4-\mathrm{QD}_{\mathrm{SA}}$ FRET pair can be calculated by

$$
R_{0}=\left(\frac{9000(\ln 10) \kappa_{\mathrm{p}}{ }^{2} Q_{\mathrm{D}}}{N_{\mathrm{A}} 128 \pi^{5} n_{\mathrm{D}}{ }^{4}} J\right)^{1 / 6}
$$

where $\kappa_{\mathrm{p}}{ }^{2}$ is $2 / 3$ for randomly oriented dipoles, ${ }^{21}$ the quantum yield for QDs is usually about 0.5 , and the refractive index $n_{\mathrm{D}}$ is 1.4 for biomolecules in aqueous solution. ${ }^{24}$ For Cy5, $\varepsilon_{\mathrm{A}}(\lambda)$ is expressed as an extinction coefficient, $\varepsilon_{\mathrm{A}}\left(\lambda_{\text {max }}\right)=\varepsilon_{\mathrm{A}}(647)=$
$250000 \mathrm{~cm}^{-1} \mathrm{M}^{-1}$. So $R_{0}$ for FRET pair QD-605/Cy5, is about $6 \mathrm{~nm}$.

Because $\mathrm{QD}_{\mathrm{SA}}$ has a broad excitation wavelength range, 488 $\mathrm{nm}$ was chosen as the excitation wavelength, minimizing the direct excitation of $\mathrm{Cy} 5$ to nearly zero by the laser source. In addition, the narrow emission spectrum of $\mathrm{QD}_{\mathrm{SA}}$ resulted in negligible crosstalk between the $\mathrm{QD}_{\mathrm{SA}}$ and $\mathrm{Cy} 5$ fluorophores. QDs and cyanine dye are among the most efficient FRET pairs used over the past decade. ${ }^{15,25,26}$ However, most of these FRET systems have generally been applied to structural study of larger biomolecules (i.e., proteins and DNA) and not small glycans. Moreover, FRET was mostly detected by imaging and microscopic techniques, which is relatively difficult to apply for quantitative analysis. The highly efficient separation power of CE for donor-acceptor complexes and the unlinked fluorophores, as well as the ability to reflect possible changes in fluorescence intensity due to the conformation changes of donor-acceptor ratio, contributes to the lower analysis uncertainty (variance) and higher FRET efficiency observed in the current study, resulting in more sensitive FRET analysis. A simple two-filter CE-LIF system for quantitative determination of disaccharide (Figure 2C) was used that could be easily extended to other FRET systems based on QDs. CE separation of donor-acceptor complexes from free donor eliminates the interference of non-FRET signal impurity. Thus, this CE-based technique possesses unique advantages of improved FRET efficiency, high sensitivity, and low sample volume requirements.

High-resolution $\mathrm{CE}$ was demonstrated to be effective in separating very small amounts of donor from FRET complex, thus solving the problem of signal impurity associated with most conventional FRET measurements. The CE-based separation of QDs and QD complexes typically shows very broad peaks. This is because earlier methods of QD synthesis had resulted in relatively broad size distribution of QDs. Improved QD synthesis has afforded commercially available QDs and derivatized QDs that are much more homogeneous in size, although some size dispersity is still present. Significant optimization of the CE separation was first required, including the selection of mobile phase, capillary type, and voltage conditions. Sodium borate buffer, the most commonly used buffer for CZE separation of QDs, afforded relatively broader peaks than those observed for sodium carbonate buffer. As the buffer $\mathrm{pH}$ was increased, the negative charge of the $\mathrm{QD}_{\mathrm{SA}}$ nanoparticles increased, whereas the net negative charge on disaccharides in the $4-\mathrm{QD}_{\mathrm{SA}}$ complex changed very little. Thus, a $\mathrm{pH}$ change from 9.0 to 11.0 both broadened peaks and increased elution time without improving resolution, making $\mathrm{pH} 9.0$ the optimal $\mathrm{pH}$ value. PEG additive afforded a sieving effect, associated with the pore size of the sieving media, improving peak resolution. However, higher PEG concentration resulted in peak broadening and poorer separations. At these higher PEG concentrations pores become too small for these large analytes to enter, decreasing resolution. The $2 \%$ PEG sieving medium concentration, selected to provide optimal performance, is in agreement with previous reports. ${ }^{20}$

In conclusion, the combination of the high extinction coefficient of the Cy5 dye and high QD quantum yield at a high ratio of dye acceptor per QD donor allows one to achieve efficient FRET with an efficiency of $92 \%$. This sensitive method can detect and quantify disaccharides at concentrations of 36 zmol, over 1000-fold more sensitive than fluorescent labeling of disaccharide analysis by CE-LIF. ${ }^{27}$ Moreover, the double 
labeling of other glycosaminoglycan-derived disaccharides and sialic acid containing glycans has been recently reported, ${ }^{4}$ making it possible to extend this detection platform to other acidic carbohydrates, including ones present in proteoglycans, glycolipids, and glycoproteins. ${ }^{24}$ Furthermore, because of the capacity of multiple binding of targets per QDs, this method can be potentially applied to oligosaccharide or polysaccharide analysis, which normally has a low FRET due to the long chain and large donor-acceptor distance.

\section{ASSOCIATED CONTENT}

\section{S Supporting Information}

Additional information as noted in text. This material is available free of charge via the Internet at http://pubs.acs.org.

\section{AUTHOR INFORMATION}

\section{Notes}

The authors declare no competing financial interest.

\section{ACKNOWLEDGMENTS}

We acknowledge funding from the U.S. National Institutes of Health in the form of Grants GM38060, HL62244, and HL096972 to R.J.L. and a fellowship from the Turkish government to E.U. and a fellowship from the Chinese government to G.L.

\section{REFERENCES}

(1) Jamieson, T.; Bakhshi, R.; Petrova, D.; Pocock, R.; Imani, M.; Seifalian, A. M. Biomaterials 2009, 28, 4717-4732.

(2) Heyduk, T. Curr. Opin. Biotechnol. 2002, 13, 292-296.

(3) Li, J. J.; Bugg, T. D. Chem. Commun. 2004, 182-183.

(4) Kwon, S.-J.; Lee, K.-B.; Solakyildirim, K.; Masuko, S.; Ly, M.; Zhang, F.; Dordick, J. S.; Linhardt, R. J. Angew. Chem. 2012, 51, $11800-11804$

(5) Truong, K.; Ikura, M. Curr. Opin. Struct. Biol. 2001, 11, 573-578.

(6) Majumdar, Z. K.; Hickerson, R.; Noller, H. F.; Clegg, R. M. J. Mol. Biol. 2005, 351, 1123-1145.

(7) Piston, D. W.; Kremers, G. J. Trends Biochem. Sci. 2007, 32, 407414.

(8) Chen, Y.; Mills, J. D.; Periasamy, A. Differentiation 2003, 71, $528-541$.

(9) Van Munster, E. B.; Gadella, T. W. J. J. Microsc. (Oxford, U. K.) 2004, 213, 29-38.

(10) Jovin, T. M.; Lidke, D. S.; Jares-Erijman, E. A. NATO Secur. Sci., Ser. B 2005, 3, 209-216.

(11) Chen, H.; Puhl, H. L.; Koushik, S. V.; Vogel, S. S.; Ikeda, S. R. Biophys. J. 2006, 91, L39-L41.

(12) Zhang, Y. H.; Zhang, H. S.; Ma, M.; Guo, X. F.; Wang, H. Appl. Surf. Sci. 2009, 255, 4747-4753.

(13) Vicente, G.; Colon, L. A. Anal. Chem. 2008, 80, 1988-1994.

(14) Song, X. T.; Li, L.; Chan, H. F.; Fang, N. H.; Ren, J. C. Electrophoresis 2006, 27, 1341-1346.

(15) Wang, J. H.; Xia, J. Anal. Chim. Acta 2012, 709, 120-127.

(16) Lo, C. K.; Paau, M. C.; Xiao, D.; Choi, M. M. Electrophoresis 2008, 29, 2330-2339.

(17) Li, Y. Q.; Wang, J. H.; Zhang, H. L.; Yang, J.; Guan, L. Y.; Chen, H.; Luo, Q. M.; Zhao, Y. D. Biosens. Bioelectron. 2010, 25, 1283-1289.

(18) Ait-Mohand, K.; Lopin-Bon, C.; Jacquinet, J. C. Carbohydr. Res. 2012, 353, 33-48.

(19) Falchi, A.; Giacomelli, G.; Porcheddu, A.; Taddei, M. Synlett 2000, 275-277.

(20) Li, Y. Q.; Wang, H. Q.; Wang, J. H.; Guan, L. Y.; Liu, B. F.; Zhao, Y. D.; Chen, H. Anal. Chim. Acta 2009, 647, 219-225.

(21) Zhang, C. Y.; Yeh, H. C.; Kuroki, M. T.; Wang, T. H. Nat. Mater. 2005, 4, 826-831.
(22) Lakowicz, J. R. Principles of Fluorescence Spectroscopy, 3rd ed.; Springer: New York, 2006.

(23) Invitrogen Inc. Qdot Streptavidin Conjugates. http://probes. invitrogen.com/media/pis/mp19000.pdf (accessed April 11, 2013).

(24) Clapp, A. R.; Medintz, I. L.; Mauro, J. M.; Fisher, B. R.; Bawendi, M. G.; Mattoussi, H. J. Am. Chem. Soc. 2004, 126, 301-310.

(25) Li, L.; Hu, X. F.; Sun, Y. M.; Zhang, X. L.; Jin, W. R. Electrochem. Commun. 2011, 13, 1174-1177.

(26) Tani, T.; Oda, M.; Sakai, H.; Araki, D.; Itoh, Y.; Ohtaki, A.; Yohda, M. J. Lumin. 2011, 131, 519-522.

(27) Chang, Y.; Yang, B.; Zhao, X.; Linhardt, R. J. Anal. Biochem. 2012, 427, 91-98. 